\title{
Microbial Epidemiology of Infectious Endocarditis in the Intravenous Drug Abuse Population: A Retrospective Study
}

\author{
William C. Lorson · R. Eric Heidel · Mahmoud A. Shorman
}

Received: November 5, 2018 / Published online: January 23, 2019

(c) The Author(s) 2019

\begin{abstract}
Introduction: The microbial epidemiology differs between infective endocarditis (IE) patients with and without a history of injection drug use. We set out to determine the prevalence and microbial epidemiology of infective endocarditis in our region, the Southeastern USA, to determine if any changes need to be made in empiric antimicrobial treatment.
\end{abstract}

Methods: The electronic medical record was reviewed for patients with IE between January 2013 and July 2017, which revealed 299 cases. The cases were then sorted between patients with and without a history of injection drug use. The growth of their initial set of blood cultures and side of cardiac involvement were then recorded. Statistical analyses were run on the data sets.

Results: There were statistically significant effects associated with both methicillin-resistant Staphylococcus aureus (MRSA) and Pseudomonas species infections occurring more often in individuals with active injection drug use, while streptococcus and enterococcus

Enhanced Digital Features To view enhanced digital features for this article go to https://doi.org/10.6084/ m9.figshare.7571036.

W. C. Lorson $(\bowtie) \cdot$ R. E. Heidel · M. A. Shorman The University of Tennessee Graduate School of Medicine, Knoxville, TN, USA

e-mail: wlorson@utmck.edu infections were more likely to occur in the population of individuals who do not inject drugs.

Conclusion: In IE patients who use or are suspected of injection drug use, first-line broadspectrum antibiotics with excellent MRSA and Pseudomonas coverage are essential.

Keywords: Injection drug use; Infectious endocarditis; Methicillin-resistant Staphylococcus aureus; Pseudomonas

\section{INTRODUCTION}

Injection drug users (IDUs) have a higher incidence of infective endocarditis (IE) compared with the general population. There are a limited number of recent studies on the microbial epidemiology of IE for IDUs and relatively none from the Southeastern USA. From the published reports, Staphylococcus aureus is considered the most common bacteria associated with IE in IDUs. Other commonly reported bacteria in IDUs include other staphylococcal and pseudomonas strains. Candida species are noted as the most common fungus [1-3]. Previous research has shown that IDUs are also at a higher risk for infections from usually nonpathogenic organisms due to using dirty needles, using tap water for dissolving the drug, or licking needles before injecting [4]. Studies have also shown that IE from injection drug use 
affects the right side of the heart significantly more often than the left [3].

Due to the extremely sparse information available on the epidemiology of this infection from the Southeastern US, we sought to determine the microbial epidemiology and the side of heart involvement of IE cases at our institution and compare this to data in published literature.

\section{METHODS}

A retrospective cohort of 299 cases of IE between January 2013 and July 2017 was analyzed, after institutional review board approval by the University of Tennessee Graduate School of Medicine Institutional Review Board (IRB reference \#4291). This study was performed in accordance with the Helsinki Declaration of 1964 and its later amendments. The IRB approved a waiver for informed consent because this was a retrospective review. The location was a large tertiary referral hospital in East Tennessee that has a referral base of East Tennessee, Western Virginia, Western North Carolina, and Southeastern Kentucky. Demographic, IV substance use status, radiographic, and echocardiographic data were collected. The blood culture results utilized were from the sets obtained in the beginning of their hospitalization. These were then recorded in a spreadsheet for analysis. Frequency statistics, chi-square analysis, and unadjusted odds ratios (ORs) with 95\% confidence intervals (95\% CIs) were used to compare independent groups. Each chart was also individually reviewed to determine whether the patient was using injection drugs by identifying a positive drug screen with a history of injection drug use, drug paraphernalia found on the person, or a patient statement of injection drug use.

\section{RESULTS}

Of the 299 cases, $184(61.5 \%)$ had findings consistent with active injection drug use, and 115 cases $(38.5 \%)$ showed no history of injection drug use. Injection drug use- and non- injection drug use-positive culture rates were $92.4 \%$ and $86.1 \%$, respectively. Methicillin-resistant $S$. aureus (MRSA) was more likely to occur in IDUs (OR 2.8, 95\% CI 1.67-4.95 $p<0.001$ ). MRSA was also the most common pathogen in IDUs and was identified in 77 of the 184 cases (42\%). Twenty-three of the 115 (20\%) cases grew MRSA in the population who did not inject drugs. Methicillin-sensitive $S$. aureus (MSSA) was the second most common bacteria for the IDUs, comprising 36 out of 184 cases $(19.6 \%)$. In contrast, there was no statistical significance found compared with the population that did not inject drugs, who had 17 MSSA cases out of 115 (14.8\%). IDUs were more likely to be infected by Pseudomonas species (OR $3.384,95 \%$ CI 1.13-10.17, $p=0.027)$, which occurred in 20 (19 Pseudomonas aeruginosa and 1 Pseudomonas fluorescens) of the 184 cases (10.9\%) compared with the population who did not inject drugs, which had 4 out of 111 (3.5\%) cases. Some bacteria were less likely to occur in IDUs, such as enterococcus infections (OR 0.32, 95\% CI 0.16-0.637, $p=0.001)$ and streptococcal infections (OR 0.384, CI 0.182-0.812, $p=0.012$, (Table 1). Other bacterial species were also identified but showed no statistical significance between the two populations (Table 2). IDUs also experienced more right-heart involvement versus left-sided involvement (OR 2.19, 95\% CI $1.29-3.74, p=0.004)$. The IDU population had 11 cases of infected prosthetic valves. The most common bacteria was MRSA, and the most common valve being affected was the prosthetic mitral valve (Table 3 ). The non-IDU population had eight cases of infected valves with the most common bacteria being enterococcus, with the

Table 1 Organisms with statistically significant differences between the population of IDUs compared the population of patients who do not inject drugs

\begin{tabular}{lllr}
\hline Organism & OR & 95\% CI & $\boldsymbol{p}$ value \\
\hline MRSA & 2.8 & $1.67-4.95$ & $<0.001$ \\
Pseudomonas & 3.38 & $1.13-10.17$ & 0.027 \\
Enterococcus & 0.32 & $0.16-0.637$ & 0.001 \\
Streptococcus & 0.384 & $0.182-0.812$ & 0.012 \\
\hline
\end{tabular}


Table 2 Organisms listed under the "other" category and number of cases involving each organism

\begin{tabular}{lll}
\hline Organism & Number of cases & IDU associated \\
\hline Brevibacterium casei & 1 & No \\
Cornybacterium & 1 & No \\
Kocuria species & 1 & No \\
Granulicatella adiacens & 2 & 1 No 1 yes \\
Legionella & 1 & No \\
Stenotrophomonas maltophilia & 3 & 2 No 1 yes \\
Enterobacter & 2 & Yes \\
Achromobacter xylosoxidans & 1 & No \\
Chryseobacterium species & 1 & No \\
Bacillus species (not anthracis) & 1 & Yes \\
Lactobacillus & 2 & 1 No 1 yes \\
Elizabethkingia meningoseptica & 1 & Yes \\
\hline
\end{tabular}

Table 3 Prosthetic valve and bacteria involved in the IDU population

\begin{tabular}{lll}
\hline Valve involved & Microorganism involved & Number of cases \\
\hline Tricuspid & Pseudomonas & 1 \\
Mitral & Candida & 1 \\
Mitral & MRSA & 2 \\
Mitral & Pseudomonas & 2 \\
Mitral & Streptococcus & 1 \\
Aortic & MRSA & 2 \\
Aortic & Streptococcus & 2 \\
\hline
\end{tabular}

prosthetic aortic valve being most commonly infected (Table 4).

\section{DISCUSSION}

S. aureus has long been associated with endocarditis in IDUs where it has been implicated in causing up to $70 \%$ of IE cases [3]. This number has surprisingly been relatively unchanged with a rate of $68.69 \%$ in the 1970 s and $65.99 \%$ in the 2000s [5]. Until recently, there have been few large studies that differentiate between MRSA and MSSA IE in IDUs. A study from San Francisco published in 2008 analyzed 184 IDUs and 63 people who did not inject drugs and showed an OR of $7.3(p<0.0001)$ for MRSA in IDUs compared with the population who did not inject drugs. This study also showed an OR of $1.95(p=0.07)$ for MSSA in IDUs, while our study did not find statistical significance between the two populations for that bacteria. Another similarity was the incidence of IE caused by enterococcus. Both the 2008 study 
Table 4 Prosthetic valve and bacteria involved in the non-IDU population

\begin{tabular}{lll}
\hline Valve involved & Microorganism involved & Number of cases \\
\hline Tricuspid & Enterococcus & 1 \\
Pulmonic & No growth & 1 \\
Aortic & Enterococcus & 2 \\
Aortic & Streptococcus & 2 \\
Aortic & MSSA & 1 \\
Mitral and aortic & Enterococcus & 1 \\
\hline
\end{tabular}

and our study showed a statistically significant lesser likelihood of IE caused by MSSA in IDUs compared with the population who did not inject drugs. Streptococci IE in IDUs was also lower than the non-injection drug use population in their study but differentiated from our study because it was not statistically significant. One of the largest differences between the two studies was that our study had 24 cases $(8 \%)$ of Pseudomonas IE, while they only had 1 case (0.004\%, Fig. 1) [6].

Our study had several cultures that exhibited no growth/sterile blood. A review article that examined literature published concerning rightsided endocarditis between January 2008 and April 2013 included a study on blood cultures obtained from 198 patients. The study showed a no-growth/sterile culture in $15.7 \%$ of their patients, which is similar to our $13.9 \%$ no-growth/ sterile culture in the population of those who did not inject drugs, but almost twice that of our IDUs (Fig. 1) [7]. It did appear from chart review that multiple patients who were in the IDU population left against medical advice from our institution, which could indicate that they had recently been seen at another institution and also left against medical advice after receiving antibiotics. This would possibly make their blood cultures appear sterile, but this is just speculation.

Suggestions for empiric coverage for endocarditis do differ slightly with sources. MRSA coverage is most often vancomycin or daptomycin [8]. While there is no true consensus on pseudomonas coverage, combination antibiotic therapy with a $\beta$-lactam and an aminoglycoside is commonly suggested $[8,9]$. Combination

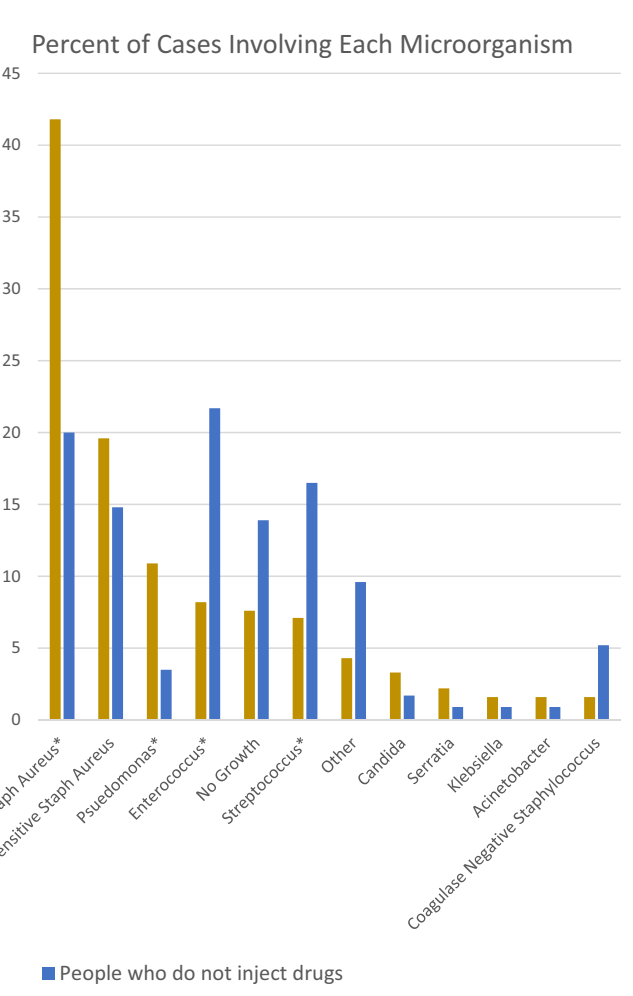

Fig. 1 Comparison of blood culture growth for IDUs and patients who do not inject drugs. ${ }^{*}$ Denotes statistical significance between IDUs and those who do not inject drugs

with a $\beta$-lactam and a fluoroquinolone is also seen in the literature [8]. A study did show promise with the use of cefepime and high-dose tobramycin, unless resistance was determined, in which meropenem and high-dose tobramycin were used [10]. Case reports and reviews have also shown survival with this combination [11]. 
It is also often thought that Pseudomonas IE occurs in outbreaks, most likely due to contamination. This is sometimes iatrogenic, but as in our case it was most likely related to the substances being injected by IDUs [12]. An example of this was associated with injection of pentazocine and tripelennamine, where specific serotypes of Pseudomonas were associated with IE in IDUs [13, 14]. During the months of October 2016-January 2017, there was an average of 1.75 cases of Pseudomonas IE in IDUs, while overall the average incidence of pseudomonas endocarditis per month of this study was 0.39 cases per month. Unfortunately, we do not have the ability to determine if the noted rise in incidence came from a specific source, such as contaminated heroin.

A limitation of this study was that it was a retrospective data collection through an electronic medical record. As a result, we could not determine the last time each patient used injection drugs, whether they were recently discharged or left against medical advice at another medical institution, or their methods of injection. Also, for a large duration of the study there was no outpatient infectious disease clinic at the medical center, which impeded our ability to follow the patients after discharge.

\section{CONCLUSION}

$S$. aureus was the most common pathogen involved with IE in IDUs followed by Pseudomonas. Data from this study solidify that in patients with confirmed or suspected injection drug use, first-line broad-spectrum antibiotics with excellent MRSA and pseudomonal coverage are essential. Extra attention for right-sided heart involvement should also be made with imaging modalities. Further studies are needed to show why our study demonstrates a significantly higher amount of IE caused by Pseudomonas than previous studies.

\section{ACKNOWLEDGEMENTS}

We would like to acknowledge Avi Das, MD, and Justin Wolfe for their assistance.
Funding. No funding or sponsorship was received for this study. The article processing charges were funded by the University of Tennessee Graduate School of Medicine.

Authorship. All named authors meet the International Committee of Medical Journal Editors (ICMJE) criteria for authorship for this article, take responsibility for the integrity of the work as a whole, and have given their approval for this version to be published.

Prior Presentation. This work was presented as an abstract with poster presentation at ID Week 2018 in San Francisco, CA, USA.

Disclosures. No outside source of funding was used for this research. William Lorson, R. Eric Heidel, and Mahmoud Shorman have nothing to disclose.

Compliance with Ethics Guidelines. All procedures performed in studies involving human participants were in accordance with The University of Tennessee Graduate School of Medicine Institutional Review Board (IRB reference \#4291) and with the 1964 Helsinki Declaration and its later amendments or comparable ethical standards. The IRB approved a waiver for informed consent because this was a retrospective review.

Data Availability. The data sets generated during and/or analyzed during the current study are not publicly available because of HIPPA compliance and privacy concerns, but are available from the corresponding author on reasonable request.

Open Access. This article is distributed under the terms of the Creative Commons Attribution-NonCommercial 4.0 International License (http://creativecommons.org/licenses/ by-nc/4.0/), which permits any noncommercial use, distribution, and reproduction in any medium, provided you give appropriate credit to the original author(s) and the source, provide a link to the Creative Commons license, and indicate if changes were made. 


\section{REFERENCES}

1. Fowler VG, Miro JM, Hoen B, et al. ICE Investigators. Staphylococcus aureus endocarditis: a consequence of medical progress. JAMA. 2005;293:3012-21. https://www.ncbi.nlm.nih.gov/ pubmed/15972563. Accessed Oct 31, 2018.

2. Crawford $\mathrm{MH}$, Doernberg S. Infective Endocarditis. In: Crawford $\mathrm{MH}$, editor. Current diagnosis and treatment: cardiology. 5th ed. New York: McGrawHill; 2017.

3. Moss R, Munt B. Injection drug use and right sided endocarditis. Heart. 2003;89(5):577-581. https:// www.ncbi.nlm.nih.gov/pmc/articles/PMC1767660/. Accessed Oct 31, 2018.

4. Miro JM, del Rio A. Infective endocarditis in intravenous drug abusers and HIV-1 infected patients. Infect Dis Clin North Am. 2002;16:273-295. https://www.ncbi.nlm.nih.gov/pubmed/12092473. Accessed Oct 31, 2018.

5. Slipczuk L, Codolosa JN, Davila CD, Romero-Corral A, Yun J, Pressman GS, Figueredo VM. Infective endocarditis epidemiology over five decades: a systematic review. PLoS One. 2013. https://doi.org/10. 1371/journal.pone.0082665.

6. Jain V, Yang MH, Kovacicova-Lezcano G, Juhle LS, Bolger AF, Winston LG. Infective endocarditis in an urban medical center: association of individual drugs with valvular involvement. $\mathrm{J}$ Infect. 2008;57(2):132-8. https://doi.org/10.1016/j.jinf. 2008.05.008.

7. Yaun S. Right sided infective endocarditis: Recent epidemiologic changes. Int $\mathrm{J}$ Clin Exp Med. 2014;7(1):199-218. https://www.ncbi.nlm.nih.gov/ pmc/articles/PMC3902260/. Accessed Oct 31, 2018.

8. Baddour LM, Wilson WR, Bayer AS, et al. American Heart Association Committee on Rheumatic Fever,
Endocarditis, and Kawasaki Disease of the Council on Cardiovascular Disease in the Young, Council on Clinical Cardiology, Council on Cardiovascular Surgery and Anesthesia, and Stroke Council Infective endocarditis in adults: diagnosis, antimicrobial therapy, and management of complications: a scientific statement for healthcare professionals from the American Heart Association. Circulation. 2015;132:1435-86.

9. Hagiya H, Tanaka T, Takimoto K, et al. Non-nosocomial healthcare-associated left-sided Pseudomonas aeruginosa endocarditis: a case report and literature review. BMC Infect Dis. 2016;16(1):431. https://doi.org/10.1186/s12879-016-1757-y.

10. Reyes MP, Ali A, Mendes RE, Biedenbach DJ. Resurgence of Pseudomonas endocarditis in Detroit, 2006-2008. Medicine (Baltimore). 2009;88: 294-301. https://doi.org/10.1097/MD.0b013e318 1b8bedc.

11. Gavin PJ, Suseno MT, Cook FV, Peterson LR, Thomson RB. Jr Left-sided endocarditis caused by Pseudomonas aeruginosa: successful treatment with meropenem and tobramycin. Diagn Microbiol Infect Dis. 2003;47:427-30. https://doi.org/10. 1016/S0732-8893(03)00135-4.

12. Seyman D, Inan D, Sepin Ozen N, Ogunc D. An outbreak of Pseudomonas aeruginosa infective endocarditis subsequent to coronary angiography. Rev Chil Infectol. 2014;31:268-73.

13. Levin MH, Weinstein RA, Nathan C, Selander RK, Ochman H, Kabins SA. Association of infection caused by Pseudomonas aeruginosa serotype O11 with intravenous abuse of pentazocine mixed with tripelennamine. J Clin Microbiol. 1984;20(4): 758-62.

14. Shekar R, Rice TW, Zierdt $\mathrm{CH}$, et al. Outbreak of endocarditis caused by Pseudomonas aeruginosa serotype $\mathrm{O} 11$ among pentazocine and tripelennamine abusers in Chicago. J Infect Dis. 1985;151:203-8. 